\title{
Muscle glycogenosis with low phosphorylase kinase activity: mutations in PHKA1, PHKG 1 or six other candidate genes explain only a minority of cases
}

\author{
Barbara Burwinkel ${ }^{1,7}$, Bin $\mathrm{Hu}^{1}$, Anja Schroers ${ }^{2}$, Paula R. Clemens ${ }^{3,8}$, Shimon W. Moses ${ }^{4}$,
} Yoon S. Shin ${ }^{5}$, Dieter Pongratz ${ }^{6}$, Matthias Vorgerd ${ }^{2}$ and Manfred W. Kilimann*,1,9

\begin{abstract}
${ }^{1}$ Institut für Physiologische Chemie, Ruhr-Universität Bochum, D-44780 Bochum, Germany; ${ }^{2}$ Neurologische Universitätsklinik Bergmannsheil, Ruhr-Universität Bochum, Bürkle-de-la-Camp-Platz 1, D-44789 Bochum, Germany; ${ }^{3}$ Department of Neurology, Mayo Clinic, Rochester, MN 55905, USA; ${ }^{4}$ Department of Pediatrics, Soroka Medical Center, Ben Gurion University of the Negev, IL-84105 Beer-Sheva, Israel; ${ }^{5}$ Stoffwechselzentrum, Dr V Haunersches Kinderspital der Universität München, D-80337 München, Germany; ${ }^{6}$ Friedrich-Baur-Institut der Universität München, Ziemssenstr. 1, D-80336 München, Germany
\end{abstract}

Muscle-specific deficiency of phosphorylase kinase (Phk) causes glycogen storage disease, clinically manifesting in exercise intolerance with early fatiguability, pain, cramps and occasionally myoglobinuria. In two patients and in a mouse mutant with muscle Phk deficiency, mutations were previously found in the muscle isoform of the Phk $\alpha$ subunit, encoded by the X-chromosomal PHKA1 gene (MIM \# 311870). No mutations have been identified in the muscle isoform of the Phk $\gamma$ subunit (PHKG 1). In the present study, we determined the structure of the $P H K G 1$ gene and characterized its relationship to several pseudogenes. In six patients with adult- or juvenile-onset muscle glycogenosis and low Phk activity, we then searched for mutations in eight candidate genes. The coding sequences of all six genes that contribute to Phk in muscle were analysed: PHKA1, PHKB, PHKG1, CALM1, CALM2 and CALM3. We also analysed the genes of the muscle isoform of glycogen phosphorylase (PYGM), of a muscle-specific regulatory subunit of the AMP-dependent protein kinase (PRKAG3), and the promoter regions of PHKA1, PHKB and PHKG1. Only in one male patient did we find a PHKA1 missense mutation (D299V) that explains the enzyme deficiency. Two patients were heterozygous for single amino-acid replacements in PHKB that are of unclear significance (Q657K and $\mathrm{Y770C}$ ). No sequence abnormalities were found in the other three patients. If these results can be generalized, only a fraction of cases with muscle glycogenosis and a biochemical diagnosis of low Phk activity are caused by coding, splice-site or promoter mutations in PHKA1, PHKG 1 or other Phk subunit genes. Most patients with this diagnosis probably are affected either by elusive mutations of Phk subunit genes or by defects in other, unidentified genes.

European Journal of Human Genetics (2003) 11, 516-526. doi:10.1038/sj.ejhg.5200996

Keywords: glycogen storage disease; phosphorylase kinase deficiency; metabolic myopathy; exercise intolerance; gene structure; pseudogene

*Correspondence: Dr MW Kilimann, Department of Cell and Molecular Biology, Uppsala University, Box 596, BMC, S-75124 Uppsala, Sweden. Tel: +49 234322 7927; Fax: +49 234321 4193;

E-mail: manfred.kilimann@icm.uu.se

${ }^{7}$ Current address: Universität Kiel and Bundesanstalt für Milchforschung, Hermann-Weigmann-Str. 1, D-24103 Kiel, Germany.

\footnotetext{
${ }^{8}$ Current address: Department of Neurology, University of Pittsburgh, Pittsburgh PA 15213, USA.

${ }^{9}$ Current address: Department of Cell and Molecular Biololgy, Uppsala University, Box 596, BMC, S-75124 Uppsala, Sweden.

Received 31 October 2002; revised 19 February 2003; accepted 21 February 2003
} 


\section{Introduction}

Disorders of glycogen metabolism (glycogenoses or glycogen storage diseases) can be caused by genetic deficiencies of various enzymes or transporters, involved either directly in the synthesis or breakdown of glycogen or in the utilization of its catabolite, glucose-1-phosphate. Glycogenoses can be multisystemic disorders or can preferentially affect only certain tissues, often but not always reflecting the tissue specificity of expression of the mutant gene. Liver and muscle are the two tissues in which glycogen is most abundant and functionally most prominent: as a reservoir for systemic glucose homeostasis in liver and for local glycolytic energy production in exercising muscle. Therefore, most glycogenoses manifest primarily in liver, in muscle, or in both, although in some forms the kidney, heart, nervous system or erythrocytes can also be affected. ${ }^{1,2}$

Phosphorylase kinase (Phk) is a regulatory protein kinase that stimulates glycogen breakdown by phosphorylating and thus activating glycogen phosphorylase. Phk deficiency alone accounts for $\sim 25 \%$ of all cases of glycogen storage diseases. Phk consists of four subunits in a hexadekameric complex, $(\alpha \beta \gamma \delta)_{4}$, and each of these subunits has isoforms or splice variants differentially expressed in different tissues. Consequently, Phk deficiency occurs in several subtypes that differ in mode of inheritance and tissue involvement. ${ }^{1,3}$

Mutations of the liver isoforms of the Phk $\alpha$ or $\gamma$ subunits (gene symbols, PHKA2 and PHKG2) cause liver-specific glycogenoses. ${ }^{4-6}$ Mutations in the ubiquitously expressed exons of the $\beta$ subunit (PHKB) cause Phk deficiency in liver and muscle, though primarily the liver is clinically affected. ${ }^{7,8}$ Liver Phk deficiency manifests in infancy or childhood with hepatomegaly and growth retardation. It is generally a benign condition whose clinical features often remit as the patients grow up. Progression to liver cirrhosis was seen only in rare cases, always in association with PHKG2 mutations. ${ }^{9,10}$

Muscle-specific glycogenosis caused by Phk deficiency, not affecting the liver, can present with three different clinical pictures. Most frequently, the disorder becomes clinically apparent at a juvenile or adult age with exercise intolerance in the form of pain, cramps, early fatigue and sometimes myoglobinuria. ${ }^{1-16}$ A late-adult onset form manifesting with slowly progressive atrophy and weakness, ${ }^{12}$ and a neonatal form with generalized muscle hypotonia and respiratory insufficiency have also been described. ${ }^{17-22}$ Three similar clinical variants are also observed in other muscle glycogenoses (myophosphorylase deficiency, phosphofructokinase deficiency), ${ }^{1,2}$ suggesting that additional genetic factors modify the clinical phenotype. Most patients with muscle-specific Phk deficiency are males, and mutations in the X-chromosomal gene of the muscle isoform of the Phk $\alpha$ subunit (PHKA1) have been identified in a mouse mutant ${ }^{23}$ and two male human patients. $^{24,25}$ However, female patients with muscle Phk deficiency have also been reported, ${ }^{13,14,17-19,21,22}$ suggestive of autosomal inheritance, and the gene of the muscle isoform of the Phk $\gamma$ subunit (PHKG1) and a musclespecifically expressed exon of the $\beta$ subunit gene, both autosomal, are additional candidates for an involvement in this disorder. To explore the possible genetic heterogeneity of muscle Phk deficiency, we have determined the structure of the human PHKG1 gene and performed a mutation analysis in PHKA1, PHKG1 and six additional candidate genes in one female and five male, unrelated patients with juvenile- or adult-onset muscle glycogenosis and low Phk activity.

\section{Subjects and methods \\ Patients}

Detailed descriptions of patients $1-3$ have been published.

Briefly, patient 1, a white American man (designated patient 2 in Clemens et $a l^{12}$ ) developed exercise-induced cramps, pain and early fatigue since the age of 6 years, and occasional pigmenturia after intense exertion. At the age of 36 years, he reported that exercise intolerance and decreasing muscle mass were slowly progressing. Muscle glycogen concentration was elevated $(2.34 \%$ wet weight; controls, $0.70 \pm 0.21 \%$ ), and subsarcolemmal glycogen accumulation was observed in muscle histology. Total phosphorylase (measured in the presence of AMP) in muscle was normal, phosphorylase- $a$ (the active form after phosphorylation by Phk, measured in the absence of AMP) was not determined. Phk activity was markedly reduced in muscle $(13 \mu \mathrm{mol} / \mathrm{min} / \mathrm{g}$ wet weight; controls, $212 \pm 80)$ but normal in red blood cells. All other enzyme activities determined in muscle were normal: debranching enzyme, phosphoglucomutase, phosphohexose isomerase, phosphofructokinase, aldolase, phosphoglycerate kinase, phosphoglycerate mutase, lactate dehydrogenase.

Patient 2, an Israeli Ashkenazi Jewish man, ${ }^{11}$ was athletic and served in a military commando unit, and developed exercise intolerance only at age 35 years. Muscle glycogen concentration was elevated (1.9\%; controls, $0.57 \pm 0.05 \%)$, and muscle histology revealed subsarcolemmal glycogen accumulation. Total phosphorylase activity in muscle was normal whereas phosphorylase- $a$ was low $(16 \mathrm{nmol} / \mathrm{min} /$ mg protein; controls, $81 \pm 16$ ). Phk activity was markedly reduced in muscle $(0.3 \mathrm{U} / \mathrm{min} / \mathrm{mg}$ protein; controls, $2.6 \pm 1.5)$ but normal in leukocytes and erythrocytes. The muscle Phk pH 6.8/8.2 activity ratio was near 1.0, both in the patient and in controls. All other enzyme activities determined in muscle were normal: $\alpha$-glucosidase, phosphofructokinase, lactate dehydrogenase.

Patient 3, a French woman, ${ }^{13}$ had experienced exertional pain, stiffness and pigmenturia since age 11, and presented at age 31 with severe exercise intolerance (pain and stiffness after climbing one floor), weakness and muscular 
atrophy. Muscle glycogen concentration was at the upper limit of the normal range (1.5\%; controls, $1.1 \pm 0.4 \%)$, and histology showed limited subsarcolemmal glycogen accumulation only in type $2 b$ fibres, which were also reduced in diameter and number. Total muscle phosphorylase was at the lower limit of the normal range $(55 \mathrm{U} / \mathrm{g}$; controls, $75 \pm 20 \mathrm{U} / \mathrm{g}$ ), whereas phosphorylase- $a$ was undetectable. Phk activity was markedly reduced in muscle $(23 \mathrm{U} / \mathrm{g}$; controls, $111 \pm 32 \mathrm{U} / \mathrm{g}$ ) but normal in erythrocytes, lymphocytes and fibroblasts. $\alpha$-Glucosidase activity and an adenylate deaminase stain were normal. Activities of glycolytic enzymes were not specifically determined, but in an in vitro anaerobic glycolysis assay, lactate formation was within the normal range with glucose-1-phosphate, glucose-6-phosphate, fructose-6-phosphate or fructose-1,6bisphosphate as substrates, but below the normal range with glycogen as the substrate, indicating a defect of glycogenolysis rather than glycolysis.

Patient 4 (HB, male, German) had led a physically very active life, serving as a bodyguard policeman, and developed exertional pain and rhabdomyolysis only after age 30 . Muscle glycogen concentration was elevated, and lightmicroscopical histology revealed subsarcolemmal vacuolar glycogen accumulation in type 1 fibres whereas type 2 fibres were reduced in diameter and number. Total muscle phosphorylase $(480 \mathrm{nmol} / \mathrm{min} / \mathrm{mg}$ protein) was at the lower limit of the normal range (500-990), whereas the phosphorylase- $a / a+b$ ratio was very low $(0.003$; normal, $0.4-0.7)$. Muscle Phk was markedly reduced $(0.27 \mathrm{nmol} /$ $\mathrm{min} / \mathrm{mg}$ protein; normal range, 2-20). All other enzyme activities determined in muscle were normal: $\alpha$-glucosidase, debranching enzyme, phosphoglucomutase, phosphohexose isomerase, phosphofructokinase, phosphoglycerate kinase, phosphoglycerate mutase, lactate dehydrogenase, $\mathrm{N}$-acetylglucosaminidase, carnitine palmitoyltransferase.

Patient 5 (JG, male, German) had also been physically very active, experiencing exertional pain and rhabdomyolysis only at age 40 . Muscle glycogen concentration was elevated (6.3\%; normal range, $0-2 \%)$, and light- and electron-microscopic histology revealed cytoplasmic subsarcolemmal vacuolar glycogen accumulation. Total phosphorylase in muscle was normal wheras the phosphorylase $a / a+b$ ratio was reduced $(0.05$; normal, $0.25-0.4)$. Phk activity in muscle $(5 \mathrm{nmol} / \mathrm{min} / \mathrm{mg}$ protein) was at the lower end of the normal range $(3-30 \mathrm{nmol} / \mathrm{min} / \mathrm{mg}$ protein). $\alpha$-Glucosidase and debranching enzyme activities and phosphofructokinase histochemistry in muscle were normal.

Patient 6 (HS, male, German), like patients 2, 4 and 5, had been athletic and highly performing in various sports activities, served in the army as a paratrooper, and developed exertional pain and rhabdomyolysis at age 25 . At age 32 he had lost $15 \mathrm{~kg}$ of weight and was incapacitated for his occupation as a scaffolding worker. Muscle glycogen concentration was elevated (3.5\%; normal, 0.6-2.0\%), and light- and electron-microscopical histology revealed cytoplasmic vacuolar, predominantly subsarcolemmal glycogen accumulation. Total muscle phosphorylase was normal whereas the phosphorylase- $a / a+b$ ratio was very low $(0.04$; normal, $0.35-0.4)$. Muscle Phk activity $(4 \mathrm{nmol} / \mathrm{min} / \mathrm{mg}$ protein) was at the lower end of the normal range (3$30 \mathrm{nmol} / \mathrm{min} / \mathrm{mg}$ protein). All other enzyme activities determined from muscle were normal: $\alpha$-glucosidase, debranching enzyme, phosphoglucomutase, phosphohexose isomerase, phosphofructokinase, phosphoglycerate kinase, phosphoglycerate mutase, lactate dehydrogenase, $\mathrm{N}$-acetylglucosaminidase.

\section{Phk activity assays}

Assays were performed in four different laboratories. Phk activity of patient 1 was determined from a frozen muscle biopsy in a two-step assay, first converting phosphorylase- $b$ to $-a$, at $\mathrm{pH} 6.8$, and subsequently measuring phosphorylase- $a$ activity (release of glucose-1-phosphate from glycogen) by an indirect UV-spectrophotometric assay. ${ }^{12}$ Phk activity of patient 2 was determined from a fresh biopsy; phosphorylase- $a$ generated by $\mathrm{Phk}$ at $\mathrm{pH} 6.8$ and $\mathrm{pH} 8.2$ was subsequently assayed by catalysing glycogen synthesis from glucose-1-phosphate and measuring the liberation of phosphate. ${ }^{11}$ Phk activity of patient 3 was determined at $\mathrm{pH} 6.8$ from a frozen biopsy according to the same principle as for patient $2 .{ }^{13,26} \mathrm{Phk}$ activities of patients 4-6 were determined from frozen biopsies at $\mathrm{pH} 8.2$ according to the same principle. ${ }^{27}$

\section{PHKG1 gene structure}

PCR primers were based on the human PHKG1 cDNA sequence (Wehner and Kilimann ${ }^{28}$ acc. no. X80590), within exon borders predicted from the mouse and rat Phkg1 gene structures. ${ }^{29,30}$ The human PHKG1 gene was amplified from genomic DNA in seven overlapping segments and sequenced, except for the interior of the longer introns (Figure 2, Table 1; GenBank accession numbers AF254249-53).

\section{Mutation analysis}

PCR reactions routinely followed a step-down temperature protocol, with the annealing temperature in the first five cycles at the theoretical primer melting temperature, five cycles at $2^{\circ} \mathrm{C}$ below, and 35 cycles at $4^{\circ} \mathrm{C}$ below. PCR products were directly cycle-sequenced and analysed on Perkin-Elmer ABI 373 or ABI 377 instruments.

The PHKA1 coding sequence ${ }^{31}$ (acc. nos. X73874, NM_002637) was amplified by RT-PCR, from total RNA that was purified either from muscle if biopsies were available (patients 1, 5, 6), or from frozen whole blood if only blood samples were available (patients 2-4). The analysed interval extended between nucleotides -65 and 3743 (including primers), in four overlapping segments. 
Table 1 Organization of the human PHKG1 gene and comparison with PHKG2 and rat Phkg1

\begin{tabular}{|c|c|c|c|c|c|c|}
\hline Exon & Size (bp) PHKG1 & cDNA position & Intron number & PHKG1 & $\begin{array}{l}\text { Intron sizes }(b p) \\
\text { PHKG2 }\end{array}$ & Phkg1(rat) \\
\hline 1 & $>84$ & -118 to -35 & 1 & 3860 & 312 & 3300 \\
\hline 2 & 117 & -34 to 83 & 2 & 1083 & 2151 & 412 \\
\hline 3 & 179 & $84-262$ & 3 & 573 & 267 & 390 \\
\hline 4 & 55 & $263-317$ & 4 & 3254 & 2082 & 4200 \\
\hline 5 & 66 & $318-383$ & 5 & 208 & 96 & 2050 \\
\hline 6 & 164 & $384-547$ & 6 & 1024 & 2600 & 860 \\
\hline 7 & 91 & $548-638$ & 7 & 116 & 94 & 144 \\
\hline 8 & 154 & 639-792 & 8 & 137 & 69 & 860 \\
\hline 9 & 126 & 793-918 & 9 & 330 & 88 & 291 \\
\hline 10 & $>318$ & 919-1236 & & & & \\
\hline
\end{tabular}

The PHKG1 CDNA sequence positions are given relative to the first nucleotide of the translation start codon. Data for the human $\gamma_{T L}($ PHKG2) and the rat $\gamma_{M}(P h k g 1)$ subunit genes are from Burwinkel et $a l^{9}$ and Cawley et $a{ }^{29}$, respectively. Exact lengths of $P H K G 1$ introns $1,2,4$ and 6 are derived from NT_031815, in agreement with approximate lengths of our PCR products.

As is known from normal controls, ${ }^{31}$ PCR products from blood included the ' $\alpha$ ' insert' (exon 19) but lack 'region $A^{\prime}$ (exon 28), which contains the main regulatory protein kinase A phosphorylation site. Conversely, again as in normal controls, PHKA1 RT-PCR products from muscle biopsies include region A but lack the $\alpha^{\prime}$ exon. The PHKA1 gene at chromosomal region Xq13 has been sequenced in the human genome project (acc. no. NT_011594) and extends in 32 exons over 132 kilobases $(\mathrm{kb})$. Exons not represented in the respective RT-PCR products were amplified, together with their flanking intron sequences, from genomic DNA (Ex19-79 to Ex19+250; Ex27-65 to Ex28+111). The PHKA1 promoter region (nucleotides -973 to +195 relative to the first coding nucleotide) was also amplified from genomic DNA of patients 2-6 and sequenced.

The human genome contains a truncated processed PHKA1 pseudogene (chromosome 1, acc. no. NT_004686) extending from 397 nucleotides upstream to 621 nucleotides downstream of the start codon equivalent (proposed pseudogene designation, PHKA1P1). This sequence has only six mismatches to the PHKA1 cDNA and is apparently transcribed at low levels because it can be amplified from blood RNA after but not before reverse transcription. Recognizable by its characteristic divergent nucleotides, it predominates in RT-PCR products from blood RNA and gives a notable background also in heart RNA, if both primers are placed within the sequence interval shared by PHKA1 and PHKA1P1. To obtain clean sequences of the $5^{\prime}$ terminal region of the authentic PHKA1 transcript from nonmuscle RNA, the reverse primer must be placed downstream of nucleotide 621 of the coding sequence.

The $P H K B$ coding sequence ${ }^{32}$ (acc. nos. X48908, NM_000293) was amplified by RT-PCR from total RNA, either from muscle if biopsies were available (patients 5+6) or else from frozen whole blood (patients 2-4). The amplified interval extended between nucleotides -12 and
3377 (including primers) in six overlapping segments. ${ }^{7}$ RTPCR products from muscle included the muscle-specific exon 26 whereas RT-PCR products from blood contained the nonmuscle exon 27 and additionally exon 2, as is known from normal controls. ${ }^{31}$ In the latter cases, exon 26 and its surrounding intron sequences $(-197$ to +124$)$ were amplified from genomic DNA. The $P H K B$ promoter region was also amplified from genomic DNA and analysed (NT_010570; nucleotides -1050 to +202 relative to the first coding nucleotide).

The PHKG1 coding sequence ${ }^{28}$ (acc. nos. X80590, NM_006213) was amplified by RT-PCR wherever muscle RNA was available (patients 1,5+6), between nucleotides 119 and 1227 in two overlapping segments. Amplification of PHKG1 mRNA from blood RNA was not possible, neither from patients nor from normal controls. If only blood samples were available, four segments of the gene comprising all coding exons and 55-125 nucleotides of their flanking intron sequences were amplified and sequenced (exons 2, 3-4, 5-6 and 7-10; exon 1 is noncoding). The promoter region (NT_031815) was amplified from genomic DNA between nucleotides -1201 and -14 (relative to the exon 1 /intron 1 junction). The presence and normal sizes of the large introns were additionally confirmed by PCRs between exons 1 and 2, exons 2 and 4, exons 4 and 6 and exons 5 and 8 (see overview in Figure 3); these long, overlapping PCR products were authenticated by terminal sequencing and analysed for size by agarose gel electrophoresis.

The CALM1, CALM2 and CALM3 mRNAs could be specifically amplified in one piece each with primers in their divergent $5^{\prime}$ - and $3^{\prime}$-UTRs, ${ }^{33}$ from either blood or muscle RNA of patients 2-6. In the CALM2 cDNA (identical to T2 of Fischer et al; ${ }^{33}$ it should be noted that the calmodulin gene numerology is inconsistent in the literature and databases) we detected six single-nucleotide differences to the published reference sequence in wobble 
positions in all five patients and several additional individuals (22 chromosomes analysed in total), suggesting sequencing errors in the published sequence.

The PYGM mRNA sequence was amplified from muscle RNA (patients 5-6) between nucleotides -46 and 2577 in three overlapping segments. The PYGM gene (20 exons) was amplified from genomic DNA (patients 2-4) in 14 segments with intronic primers, and sequences of exons and flanking intron regions were compared with the revised genomic sequence of PYGM. ${ }^{34,35}$

The 13 exons and their flanking intron sequences of the PRKAG3 gene (NT_005289) were amplified in eight intervals from genomic DNA of patients 2-6.

\section{Results}

\section{PHKA1 mutation in only one patient out of six}

All six patients presented with exercise intolerance and an onset of symptoms in childhood or adult age. They were analysed for mutations in the PHKA1 coding sequence by RT-PCR and direct sequencing. A sequence abnormality was only found in patient 1 . He carries an $A$ to $T$ transversion in codon 299, giving rise to the replacement of aspartate by valine (D299V). The affected Asp 299 residue is, within a highly conserved sequence context, identical in the Phk $\alpha_{\mathrm{M}}, \alpha_{\mathrm{L}}$ and $\beta$ subunits of several species (Figure 1). Moreover, the corresponding residue in the liver isoform of the $\alpha$ subunit (PHKA2) was also mutated in a patient with liver Phk deficiency (D299G missense mutation). ${ }^{36}$ The PHKA1 promoter region was additionally analysed from patients $2-6$, but no sequence abnormalities were found.

\section{No mutations in PHKG1 or in the muscle-specific exon 26 of $P H K B$}

All six patients were analysed for PHKG1 mutations by amplification and sequencing of mRNA (patients 1,5 and 6) or genomic DNA (patients 2-4). The PHKG1 promoter region was sequenced from patients 2-6. PCR products of normal lengths were obtained from all. No sequence abnormalities were found. Single-nucleotide polymorphisms in wobble positions of four codons, in intron 7 and in the promoter region occurred in various combinations in these patients and in additional individuals, none of which correlated with muscle glycogenosis (see paragraph on PHKG1 gene structure below).

To probe for large-scale rearrangements of the PHKG1 gene, PCRs that individually bridge the large introns 1,2 , 4 and 6 were additionally performed. Together with the sequenced PCR products they covered the entire gene in overlapping fashion (Figure 3). Amplification products of normal lengths were obtained from all six patients and several controls (not shown).

From all patients, $P H K B$ exon 26 was either amplified and sequenced by RT-PCR in the context of the muscle

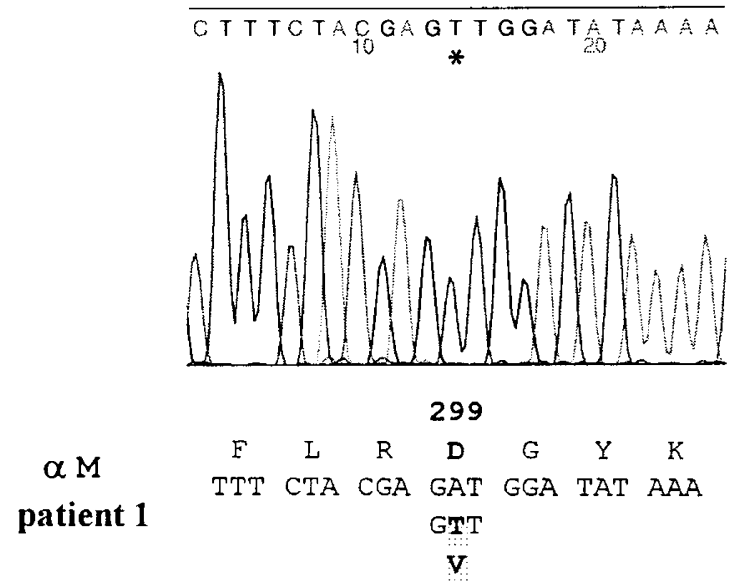

\begin{tabular}{|c|c|c|c|c|c|c|c|c|c|c|c|c|c|c|c|}
\hline $\mathrm{H} \propto \mathrm{M}$ & 290 & G & C & C & $R$ & $E$ & I & $R$ & D & G & $Y$ & K & $\mathrm{T}$ & $\mathrm{p}$ & K \\
\hline$\alpha M$ & 290 & G & C & C & R & $F$ & I & $R$ & D & G & $Y$ & K & $\mathrm{T}$ & $\mathrm{P}$ & $K$ \\
\hline$\alpha L$ & 290 & G & C & C & $\mathrm{R}$ & $E$ & I & $\mathrm{R}$ & D & G & $Y$ & K & $\mathrm{T}$ & P & R \\
\hline$\alpha L$ & 290 & G & C & C & $R$ & $E$ & I & $\mathrm{R}$ & D & G & $Y$ & $K$ & $\mathrm{~T}$ & $P$ & $\mathrm{R}$ \\
\hline$\beta$ & 326 & G & $F$ & K & $\mathrm{R}$ & $F$ & $\mathrm{~L}$ & $R$ & D & G & $Y$ & & $\mathrm{~T}$ & $S$ & I \\
\hline $\mathrm{R} \beta$ & 326 & G & $E$ & K & $\mathrm{R}$ & $\mathrm{E}$ & $\mathrm{L}$ & $\mathrm{R}$ & D & G & $Y$ & $R$ & $T$ & S & $\mathrm{L}$ \\
\hline
\end{tabular}

Figure 1 Hemizygous missense mutation in the $X$-chromosomal PHKA1 gene of patient 1. A sequencer tracing is shown at the top, with an asterisk marking the mutant position. The sequence alignment at the bottom illustrates the high conservation of Asp 299 and its vicinity in the human and rabbit $\alpha_{\mathrm{M}}, \alpha_{\mathrm{L}}$ and $\beta$ subunit sequences.

mRNA sequence, or amplified together with its flanking sequences from genomic DNA and analysed. However, no abnormalities were found in any of these sequences.

Heterozygous $P H K B$ amino-acid replacements in two out of five patients

Having explored all muscle-specifically expressed Phk coding sequences (full-length PHKA1, full-length PHKG1 and exon 26 of $P H K B$ ), we analysed the remaining $P H K B$ coding sequence based on the following considerations. 30 out of 33 exons of the single $P H K B$ gene are constitutively expressed in all tissues (exon 2 is expressed in brain and other nonmuscle tissues but not in muscle). A total of 10 different mutations (all but one truncating mutations) have been identified in these sequences, giving rise to a ubiquitous Phk deficiency clinically dominated by liver involvement whereas muscle is largely asymptomatic. Mutations in these constitutively expressed $P H K B$ sequences would therefore normally be expected to yield a liver rather than a muscle phenotype. In the liver, however, the Phk $\beta$ subunit forms a holoenzyme complex with liver isoforms of the $\alpha$ and $\gamma$ subunits which interacts with the liver isoform of its substrate protein, glycogen phosphorylase, whereas in muscle it interacts with the corresponding muscle isoforms. It is conceivable, therefore, that missense mutations at certain positions might selectively perturb the interaction of the $\beta$ subunit only with the 
muscle but not the liver isoform (or vice versa) of one of its partner polypeptides. Precedents for unexpected cell-type specificity of Phk deficiency have been found associated with missense mutations at certain positions in the $\alpha_{\mathrm{L}}$ subunit ${ }^{4,5,36}$ and also, in a single case, in the $\beta$ subunit. ${ }^{37}$ Also mutations in ubiquitously expressed sequences of the debranching and branching enzymes can give rise to glycogen storage diseases of unanticipated tissue specificities. ${ }^{38-40}$

In two of the five remaining patients, we found heterozygosity for a single amino-acid replacement each, both present in the mRNA in similar abundance as the normal sequence. Patient 4 was heterozygous for an $A>G$ transition replacing tyrosine 770 by cysteine. Tyrosine 770 is absolutely conserved between the $\alpha_{\mathrm{M}}, \alpha_{\mathrm{L}}$ and $\beta$ subunit sequences of multiple species. It is immediately adjacent to the high-affinity calmodulin binding site of the muscle splicing variant of the $\beta$ subunit that comprises amino acids 771-795. Patient 6 was heterozygous for a $C>A$ transversion replacing glutamine 657 by lysine. Glutamine 657 is conserved between the human, rabbit, mouse, dog and chicken $\beta$ subunits. Similarity with the $\alpha$ subunits in this sequence region is low, but the RLQT motif of which glutamine 657 is part may correspond to an RIQT/RVQL motif present in a colinear position in the $\alpha_{M}$ and $\alpha_{L}$ subunits, respectively. The same $P H K B$ nucleotide residue has been found mutated in two unrelated patients with liver Phk deficiency (there, however, to a T residue creating a stop codon), ${ }^{7}$ suggesting that it may be hypermutable. Neither of these two single-nucleotide replacements was found in 52 additional $P H K B$ chromosomes that we have analysed to date by sequencing. In Vanden Berg et al, ${ }^{8}$ however, the Y770C variant was observed in three out of 82 control chromosomes.

Promoter mutations interfering with binding of a transcription factor important for the much higher expression of $P H K B$ in muscle than in other tissues ${ }^{41}$ could also cause an essentially muscle-specific Phk deficiency. However, no sequence abnormalities were found in the $P H K B$ promoter regions of patients 2-6.

\section{No mutations in the calmodulin genes CALM1, CALM2 and CALM3}

Having analysed all coding sequences of Phk-specific subunits that are expressed in muscle, without finding clear deficiency mutations in five out of six patients, we turned to the subunit $\delta$, calmodulin. Calmodulin is probably expressed in all cell types. Moreover, it is produced by three different genes each of which is also expressed in multiple tissues, though at different relative levels. ${ }^{42}$ Therefore, a mutation in any individual calmodulin gene is expected to create neither a total nor a tissuespecific deficiency of calmodulin and is anticipated to affect, if at all, multiple calmodulin-interacting proteins and not only Phk. Phenotypes neither of natural nor of man-made calmodulin mutations in vertebrates have as yet been reported. However, Phk is very abundant in skeletal muscle and sequesters half of the total calmodulin in this tissue, and there is a regulatory interplay between the expression of calmodulin and of the other Phk subunits in muscle. ${ }^{43}$ Depending on the competition between Phk and other calmodulin-binding proteins for limiting amounts of calmodulin, it is therefore conceivable that a calmodulin mutation could primarily manifest as a Phk-deficient muscle glycogenosis.

The coding sequences of all three calmodulin mRNAs were analysed from patients 2-6, but no sequence abnormalities were found.

\section{No mutations in the PYGM and PRKAG3 genes}

The muscle isoform of glycogen phosphorylase (PYGM) is the substrate of muscle Phk, and it is conceivable that missense mutations of amino-acid residues participating in the conversion of PYGM from the inactive to the active conformation by phosphorylation could give rise to low activity of phosphorylase- $a$ but normal activity of total phosphorylase. AMP-dependent protein kinase (PRKA) has an important regulatory role in energy metabolism and may affect, directly or indirectly, also Phk activity. Mutations in the muscle-specific regulatory $\gamma 3$ subunit, PRKAG3, were recently found to cause a mild muscle glycogenosis in pigs. ${ }^{44}$ Therefore, we also analysed the PYGM and PRKAG3 genes of patients $2-6$. However, no sequence abnormalities were found.

\section{PHKG1 gene structure}

As a prerequisite for the mutation analysis described above, we determined the structure of the human PHKG1 gene (see Methods). The gene consists of 10 exons and extends over $\sim 12 \mathrm{~kb}$, thus having a similar size and the same exon/ intron architecture as the human PHKG2 and the rodent Phkg1 genes (Figures 2 and 3, Table 1). Besides a few polymorphic nucleotides, this gene sequence is 100\% identical to the cDNA. This indicates that we have analysed the authentic, expressed PHKG1 gene rather than pseudogene sequences (see below).

Sequencing of PCR products from multiple individuals in the course of our studies identified four single-nucleotide polymorphisms in wobble positions of codons Cys185 (TGC>TGT; allele frequency, one (patient 5) out of 28 chromosomes analysed), Pro228 (CCG > CCA; one (patient 1) out of 28 chromosomes), Tyr277 (TAC>TAT; 6/28), Val293 (GTG > GTA; 6/28), one polymorphism at nucleotide nine of intron 7 ( $\mathrm{a}>\mathrm{g} ; 8 / 14)$, and two in the promoter region (-593: TCC $[\mathrm{C}>\mathrm{T}] \mathrm{G}$; nine out of 16 chromosomes analysed) and (-449: AAAG[T $>$ C]CT; 11/16). In all 16 chromosomes analysed, an additional $\mathrm{C}$ residue was found inserted before nucleotide -916 of the promoter reference sequence (GCCACC), suggesting a sequencing error in the latter. The 831T(Tyr277) and 879T(Val293) variants always 
Exon 1 GCCTTCAGCCCTCTGTGGTCCCCTCTCCCCGGGGGGCTTTGGGATTCT TGTCAAGCTCCT TCAAGAGCC TGCAAGCACT TAACCgt gagtagctagcCCagt gat ggacaaaagCCaggt gataggcagggagcggggaggactgggggtggagaagatagaaacccccacatccat tccatccatgtctcttttct gaaggcggat ggaccagggagagtggeagcat tgaccett gaa

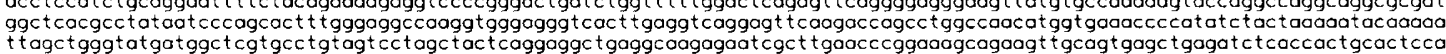

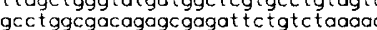

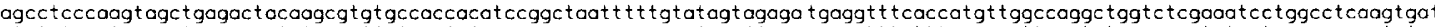

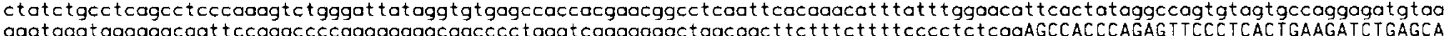

Exon 2

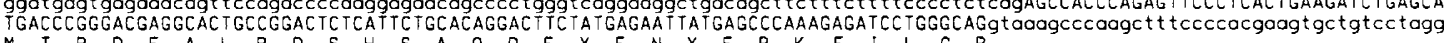
$M T T R$ D E A L P D S H S A Q D F Y E N Y E P K E I L G R

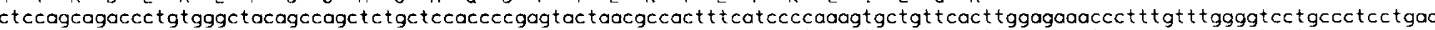

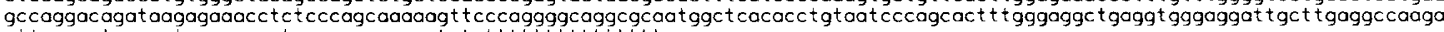

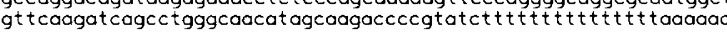

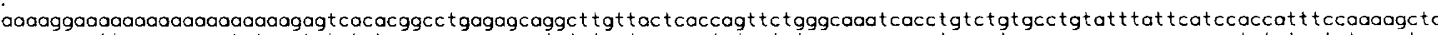
agaggaagt tgggggaaag t taaat ctct gt caagggcagga catctct gct gcagcctctaatct gggaccccccat ggggtgaggggccccccgaggccatct gtcctct gcactc

Exon 3 CagGGGCGTTAGCAGTGTGGTCAGGCGATGCATCCACAAGCCCACGAGCCAGGAGTACGCCGTGAAGGTCATCGACGTCACCGGTGGAGGCAGCTTCAGCCCGGAGGAGGTGCGGGAGC

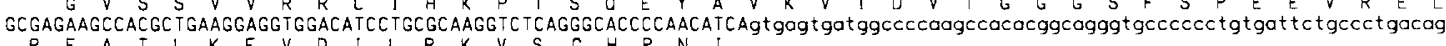

cccaagccagggagt gt ggccccaccgcacagccoactagaaccacccaggct caaggctcccagctccaccctcact get ct ga tagaaggggtcccaggcagggat ggat gcaggcag

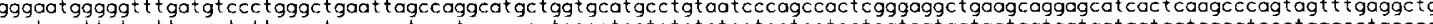
cagtgat

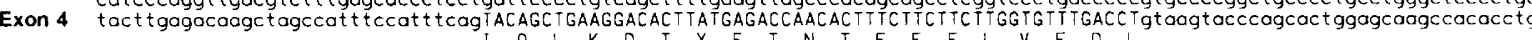

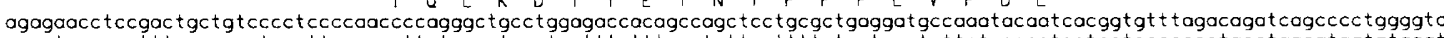
agget gccgget t ggaaacctgggt tcaccact tgtgggetggatcatt tct taaatct tggtt t tctcat cagtat tat gggagt gat aatagcacagtgactggcatagtgtgaat

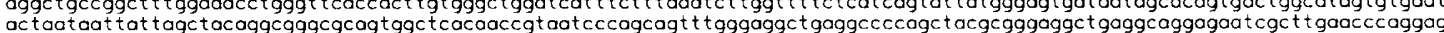
geggaggt tgaagt gagecgagat cacgecgt tgaact ccagect ggge tacgagagcgaaac tcco

cctcatgaaatggt gggaaaaat tt t tccaggggaaaaot tcagtcaggggagaaacctaggectat tcctcggagggt gat gagagcct gagcccctgat gtgggccacetct ccage ctcctctgctt tetcccagttct tccagaacact tctcact tccccggctcctctccccagactgtggt tctccacggagagtggcccact tagct tc tagaggtatcagggcggaagg

Exon 5 cagctctctgggagcagggtaaggcagtggtggtet tgggaccgatccagctatcaaccetctgtt teCacagGATGAAGAGAGGGGAGCTCT T TGACTACCTCAC TGAGAAGG CAC TTGAGTGAGAAGGAACCAGgtgagggtccctt tgccettctctccgctccctcccacgtggct tagggccacaggcactggggtctcaggggaggcagggcgtgcacatgtct ggget

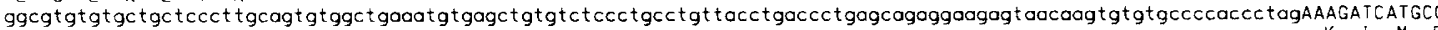

Exon 6 AGCTCTGCTGGAGGTGATCTGCACCTTGCACAAACTCAACATCGTGCACCGGGACCTGAaGCCCGAGAACATTCTCTTGGATGACAACATGAACATCAAGCTCACAGACT T TGGCTITIC

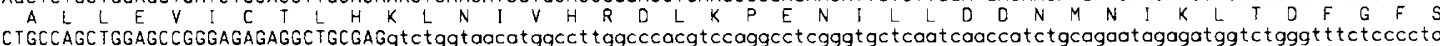

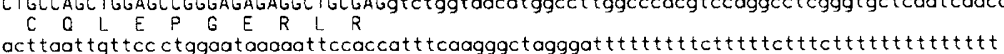

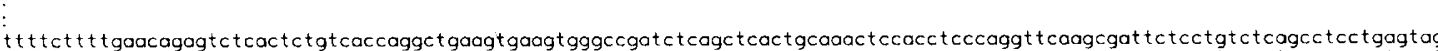

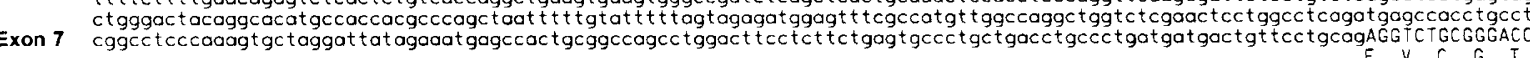
CCCAGT TACCTGGCCCCTGAGATTATCGAGTGCTCCATGAATGAGGACCACCCGGGCTACGGGAAAGAGGTGGACATgtgagtgcaccgggaaoggaggagcaccCagc taggC tgggCO

Exon 8 agggaggaagtaggcaggget gggccggtgcccetggegctgggtaagtggccet gggece tegcatgtccagGTGGAGCACTGGCGTCATCATGTACACGC TGCTGGCCGGC TCCCCGC CCT TCTGGCACCGGAAGCAGATGCTGATGCTGAGGATGATCATGAGCGGCAACTACCAGTTTGGCTCGCCCGAGTGGGATGATTACTCGGACACCGTGAAGGACCTGgtgagaggCCOg

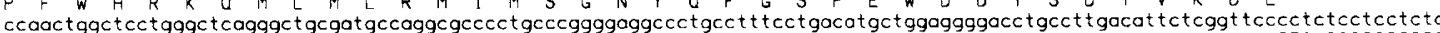

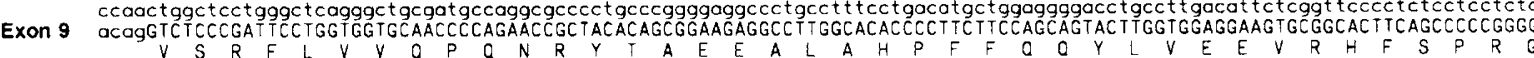
GAAGTTCAAGgtactaagcgtcctgatccaggcecgccagetccctaagtgcaaacctctaagccccetcctctcccaggagtect tacacccegtgaat ttccagagtacccatccct GAAGT K

ccctcccagctgecctgtgat gget tcaaagacggcaggtgtcagcccact gget cggtccctgaggat get ggecaagt gggaagt gggggacact aggaaggcccc gcagaagccace

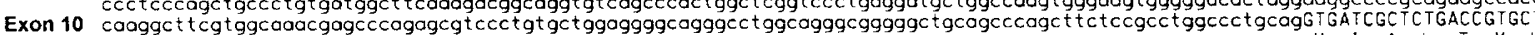
GGCTTCAGTGCGGATCTACTACCAGTACCGCCGgGTGAAGCCTGTGACCCGGGAGATCGTCATCCGAGACCCCTATGCCCTCCGGCCTCTGCGCCGGCTCATCGACGCCTACGCTTICCC A

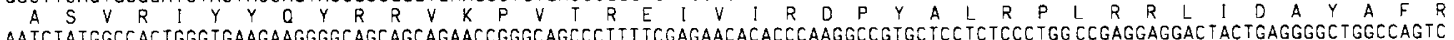
AATCTATGGCCACTGGGTGAAGAAGGGGCAGCAGCAGAACCGGGCAGCCCTTTTCGAGAACACACCCAAGGCCGTGCTCCTCTCCCTGG CCGAGGAGGACTACTGAGGGGCTGGCCAGTC AGGGAGGGCTAGGGGGCAGGTGGGGAGGGGAGCCATGGAAATACAAGTCAAAGGGGT

Figure 2 Sequence of the human PHKG1 gene. Exon sequences are in uppercase letters and introns in lowercase letters. Aminoacid sequences are given below exons. The sequence was determined in five contigs, with gaps in introns $1,2,4$ and 6. Nucleotide numbering is separate for each contig whereas amino-acid numbering is contiguous. These PHKG1 sequences are available under GenBank accession numbers AF254249-53.

occurred in linkage with each other (both lie in exon 9) but were found in linkage with either variant of the intron 7 polymorphism.

Several PHKG1-related genomic sequences have recently emerged from the Human Genome Sequencing Project (Figure 3). One of them is identical to the cDNA and gene sequences determined and analysed for mutations by us and therefore represents the authentic, expressed PHKG1. Our analysis of the PHKG1 promoter region for mutations is based on this sequence. At least three additional, heavily rearranged pseudogene sequences exist in the human genome, designated PHKG1P1-P3 by us (Figure 3). With multiple small nucleotide replacements, insertions and deletions throughout exons and introns they have $~ 90 \%$ sequence identity with $P H K G 1$ but are more similar among each other.

These pseudogenes do not appear to be transcribed. No corresponding ESTs can be found in the sequence database, they were not amplifiable from blood RNA (unlike the PHKA1P1 pseudogene transcript: see Methods section on 

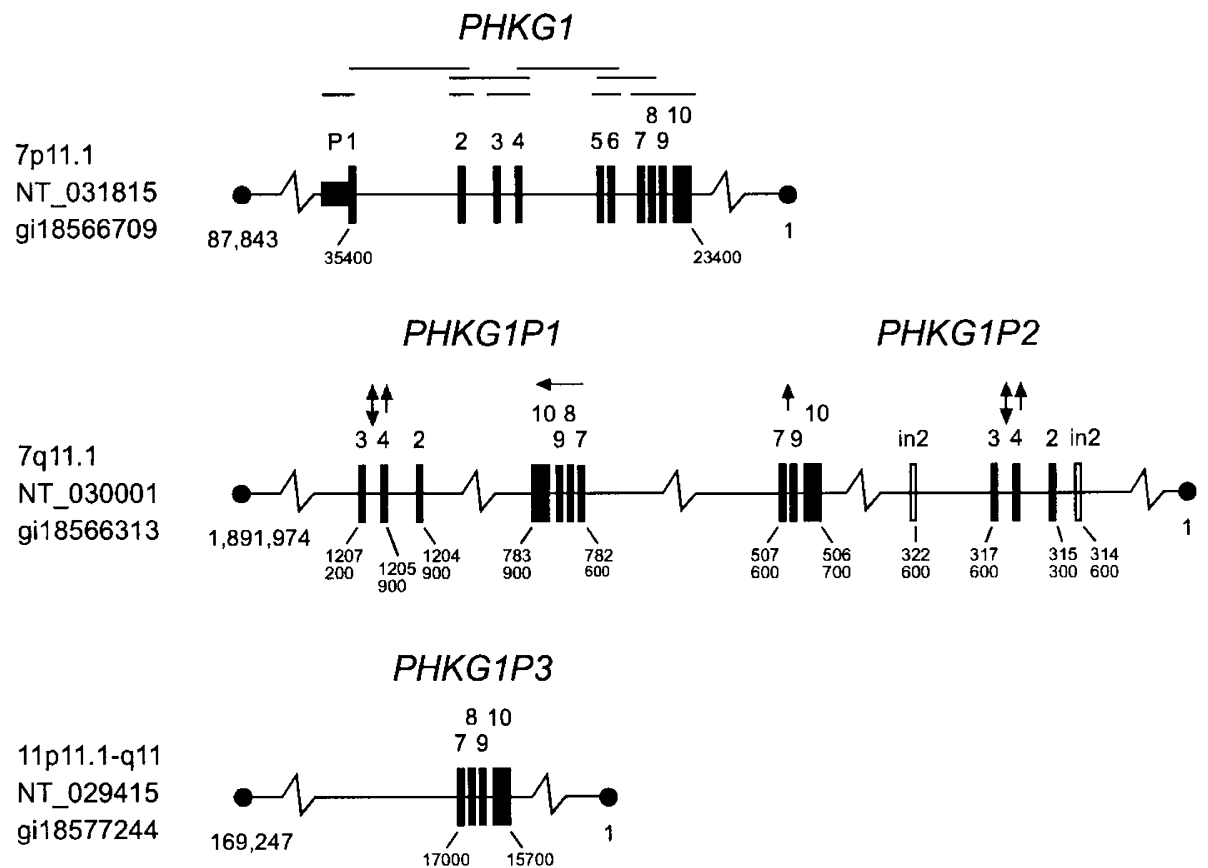

Figure 3 Schematic organization of PHKG1 and three PHKG1-related pseudogenes. Identifiers of Human Genome Sequencing Project contigs and tentative chromosomal localizations are given at the left. Exons are numbered above, and nucleotide numbering of the contig ends and of selected motifs within the genes are indicated below. The narrow box to the left of PHKG 1 exon 1 indicates the promoter region (P). Horizontal lines above PHKG1 indicate the sequence intervals amplified from the genomic DNA of patients and additional controls and analysed for mutations by full-length sequencing (short intervals in the lower row) or for normal size by agarose gel electrophoresis (long overlapping intervals in the upper row). Note that portions of the rearranged PHKG1P1 and PHKG1P2 pseudogenes are separated by intervals of several hundred kilobases and exon order is partially perturbed. All exons are shown in sense orientation with the exception of the inverted block of exons 7-10 in PHKG1P1 (horizontal arrow above). Upward-pointing arrows indicate deletions (part of exon 4 and the beginning of intron 4 in PHKG1P1 and -P2; part of exon 7, intron 7, exon 8 and the beginning of intron 8 in PHKG1P2). Double arrows above intron 3 of PHKG1P1 and $-P 2$ indicate that part of intron 3 including a $(T A A)_{15}$ repeat is replaced by an unrelated sequence.

PHKA1 mutation analysis), and sequences of PHKG1 RTPCR products from muscle and heart had no background of the characteristic pseudogene sequence deviations. PCR of genomic DNA may coamplify PHKG1 and pseudogene sequences where they are colinear (exon 2 and exons 710). However, we could specifically amplify PHKG1 with primers placed on mismatches or into regions of pseudogene rearrangements.

ESTs from the contigs harbouring PHKG1 and its pseudogenes have been mapped to centromere-near regions of human chromosomes 7 and 11 (Figure 3), in accordance with previous in situ hybridization of human chromosomes with the rabbit Phkg1 cDNA probe. ${ }^{45}$ However, genetic mapping of mouse Phkg1 to chromosome 5 employing polymorphic short tandem repeats within the authentic Phkg1 gene sequence, together with considerations of known synteny with neighboring genes, suggested $7 q 21-q 22$ as the most likely localization of human PHKG1. ${ }^{46}$ This would also be in accordance with an additional weaker in situ hybridization signal at the latter position. ${ }^{45}$ The definitive chromosomal localization of the bona fide PHKG1 gene, its neighbouring genes and pseudogenes may require additional, more discriminative investigation.

\section{Discussion}

Our mutation search covered all coding sequences that contribute to the expression of Phk in muscle: the musclespecifically expressed PHKA1, PHKG1 and $P H K B$ exon 26 sequences; the ubiquitously expressed $P H K B$ sequences; and also the three calmodulin genes that give rise to the Phk $\delta$ subunit. We also analysed the PHKA1, PHKB and $P H K G 1$ promoter regions over lengths of $\sim 1 \mathrm{~kb}$ upstream of the translation start sites and, as additional candidates that might indirectly give rise to low phosphorylase- $a$ or Phk activities, the PYGM and PRKAG3 genes. Splice site mutations would also have been detected, either directly through sequencing of the exon-flanking genomic DNA regions or indirectly, by RT-PCR of the mRNAs. The Phk liver isoform genes, PHKA2 and $P H K G 2$, were ruled out because in muscle they are expressed only in minute amounts compared to the respective muscle isoforms, $^{41,47,48}$ and individuals with PHKA2 or PHKG2 
mutations have normal Phk activity in muscle and do not exhibit exercise intolerance. ${ }^{4,6,9}$

Only a single mutation was identified among six patients: a PHKA1 missense mutation in a male patient (D299V). It affects a highly conserved amino-acid residue, and the corresponding residue in the PHKA2 gene has also been found mutated in a patient with liver Phk deficiency, confirming that it is functionally important. After one nonsense and one splice-site mutation, this is only the third human PHKA1 mutation identified, confirming that PHKA1 mutations cause Phk-deficient muscle glycogenosis. Whereas the first PHKA1 mutation was associated with the late-adult atrophic phenotype, the second and the present mutation represent the juvenile-onset type with exercise intolerance.

If the present results can be generalized, they suggest that most cases diagnosed biochemically with musclespecific glycogenosis and low Phk activity cannot be explained by coding sequence, splice-site or promoter mutations in PHKA1, nor indeed in any of the other genes encoding Phk subunits in muscle. We have additionally analysed two infantile cases of severe muscular hypotonia with muscle glycogenosis and low Phk or phosphorylase- $a$ activities without finding mutations: an unpublished case (FH) which we analysed in PHKA1, PHKG1, PHKB, CALM1, CALM2 and CALM3, and a published case ${ }^{22}$ which we analysed in $P H K G 1$. It may be significant that also from 10 muscle Phk deficiency cases described by others, ${ }^{14}$ only one PHKA1 mutation has been reported. ${ }^{25}$ PHKG1 remains the last Phk gene in which no mutations have been identified.

The occurrence of heterozygous $P H K B$ amino-acid replacements in two of the five remaining patients is intriguing, but it is unclear whether they contribute to the low Phk activity and muscle disease of their carriers. In a dominantnegative fashion, the mutant polypeptides may perturb assembly, stability or regulatory properties of the holoenzyme. They may do so tissue-specifically by selectively perturbing interaction with the muscle isoforms but not the liver isoforms of the $\alpha$ or $\gamma$ subunits, or by being sensitive to specific aspects of the intracellular milieu of muscle such as protein turnover, neural or endocrine regulation, or exercise-induced $\mathrm{pH}$ fluctuation. Neither of the two patients has a family history of muscle disease to support a dominant mode of inheritance, nor were the three control individuals that were found heterozygous for the Tyr770Cys replacement in Van den Berg et $a l^{8}$ reported to have muscle disease. However, muscle Phk deficiency may be of low clinical penetrance. Four of our six patients developed symptoms only in mid-adult age after being physically very active for many years (and perhaps only for this reason), and none of the 11 known patients with combined Phk deficiency in liver and muscle because of truncating $P H K B$ mutations has developed similar muscle disease. It is also possible that the
PHKB amino-acid replacements found here predispose for the manifestation of muscle disease in combination with another genetic defect. ${ }^{49}$ These possibilities cannot be tested in vitro and the small number of cases allows no statistically valid conclusion. The analysis of more muscle glycogenosis cases may clarify whether these or other $P H K B$ sequence variants are indeed enriched in this patient population.

Our five unsolved cases may have mutations in DNA sequences that were not covered by our analysis, for example, in remote transcription control elements. The available samples did not allow quantification of Phk subunit mRNAs or proteins in muscle from any of these patients. The existence of several PHKG1 pseudogenes indicates that PHKG1 has undergone repeated large-scale recombinations during evolution, involving in particular the long introns 1, 2, 4 and 6. However, we did not find any indications of rearrangements of the transcribed PHKG1 gene in our patients, by systematic amplification in multiple overlapping segments (Figure 3). Intact exons 1, 3-6 and the promoter region, unique to PHKG1, could be amplified from all, and mRNAs of normal sequence are expressed in muscle of patients 5 and 6 .

Phk expression or activity might be downregulated in response to a buildup of glucose phosphates and glycogen caused by a primary defect of another protein involved in muscle glycogen or glucose catabolism. For example, low liver Phk activities were determined in a family with hepatorenal glycogenosis (Fanconi-Bickel syndrome) with a mutation in the GLUT2 glucose permease but not in Phk subunits. ${ }^{50}$ Several cases with concurrent low activities of Phk and phosphofructokinase or debranching enzyme have also been described. ${ }^{17,19}$ Three of our six patients, however, were analysed for activities of other multiple enzymes known to cause glycogen storage disease (see Subjects and methods) and all activities were normal. A primary deficiency of one of the remaining glycolytic enzymes not analysed or, for example, of a transporter mediating the release of lactate or alanine from glycolytically active muscle, ${ }^{51,52}$ remains possible. Mutations in transcription factors acting on Phk subunit genes (cf. the impact of C/EBP isoforms on glycogen metabolism), ${ }^{53,54}$ in scaffolding proteins that assemble glycogen particles with their metabolizing and regulatory enzymes including Phk (Lerin et $a l^{55}$ ), or in one of the many proteins involved in the transmission of regulatory signals to Phk such as protein kinase A, might also affect the expression, the stability or the activity regulation of Phk.

Finally, as Phk is an unstable enzyme, the biochemical diagnosis of Phk deficiency in muscle may be particularly error-prone under clinical conditions. In our previous work, the large majority of cases with a biochemical diagnosis of liver Phk deficiency could be successfully ascribed to mutations affecting the coding sequences of one of the candidate genes (PHKA2, PHKB or PHKG2), showing 
that the biochemical diagnosis of Phk deficiency from liver or red blood cells is usually reliable. Also, in all our unsolved muscle cases elevated glycogen is documented both by morphological and biochemical analysis and the low Phk activities are corroborated by low phosphorylase- $a$ activities. However, in muscle tissue the Phk and phosphorylase- $a$ activities may be more labile (eg, through dephosphorylation or proteolysis) than other enzymes that were normal in the biopsies analysed here. Phk and phosphorylase- $a$ activities may suffer before biopsy (sampling from acutely or chronically damaged muscle) or after (delayed or slow freezing of tissue specimens, insufficient inhibition of phosphatases or proteases after homogenization) (I Maire, personal communication). In rat muscle, Phk activity and protein levels have been shown to drop deeply following experimental degeneration ${ }^{56}$ or denervation. 57

To avoid a biochemical misdiagnosis of muscle Phk deficiency, special care may be necessary in the timing, site choice and specimen handling of muscle biopsies, and other enzyme deficiencies that can cause a similar glycogenosis should be comprehensively excluded even if low Phk and phosphorylase- $a$ activities are measured. It may also be advisable to assay Phk routinely at $\mathrm{pH} 6.8$ and 8.2 , because Phk activity is markedly $\mathrm{pH}$-dependent and the $\mathrm{pH}$ 6.8/8.2 activity ratio reflects the enzyme's molecular status. Dephosphorylation will reduce the $\mathrm{pH}$ 6.8/8.2 activity and slight proteolysis will raise it, while activity loss at high $\mathrm{pH}$ will be indicative of a loss of total Phk through heavy proteolysis, denaturation, or reduced biosynthesis.

In conclusion, in most of our patients with a musclespecific glycogenosis and low activities of $\mathrm{Phk}$ and phosphorylase- $a$, genetic defects could not be detected in PHKA1, PHKG1, PYGM or five other candidate genes. It is unclear to what extent elusive Phk subunit mutations, Phk downregulation secondary to other molecular abnormalities, or unspecific prebiopsy or artifactual postbiopsy activity losses contribute to this patient population. We suspect that in general, a high percentage of biochemically diagnosed 'muscle Phk deficiency' cases will be negative for conventional mutations in Phk subunits. High-quality muscle biopsies and a continuing search for alterations in multiple enzyme activities, protein levels or mRNA levels will be needed to resolve this group.

\section{Acknowledgements}

We thank the patients, their primary care physicians (Drs Vincensini, Kerschbaumer, Ormanns), and Professor Andrew G Engel (Mayo Clinic, Rochester, MN) and Professor Henri Carrier (Lyon) for their support, and Dr Irene Maire (Centre Hospitalier Universitaire, Lyon) for critical reading of the manuscript and valuable suggestions. This work was supported by the Deutsche Forschungsgemeinschaft (Ki 324/11), the University of Bochum Medical School intramural research funding program (FoRUM), and the Fonds der Chemischen Industrie.

\section{References}

1 Chen YT: Glycogen storage diseases, in Scriver CR, Beaudet AL, Sly WS, Valle D (eds): The Metabolic and Molecular Bases of Inherited Disease, 8th edn. New York: McGraw-Hill, 2001, pp. 1521-1551.

2 Tsujino S, Nonaka I, DiMauro S: Glycogen storage myopathies. Neurol Clin 2000; 18: 125-150.

3 Kilimann MW: Glycogen storage disease due to phosphorylase kinase deficiency, in Swallow DM, Edwards YH (eds): Protein dysfunction in human genetic disease, Oxford: BIOS Scientific Publishers, 1997, pp 57-75.

4 Burwinkel B, Amat L, Gray RGF et al: Variability of biochemical and clinical phenotype in X-linked liver glycogenosis with mutations in the phosphorylase kinase PHKA2 gene. Hum Genet 1998; 102: 423-429.

5 Hendrickx J, Lee P, Keating JPet al: Complete genomic structure and mutational spectrum of PHKA2 in patients with X-linked liver glycogenosis type I and II. Am J Hum Genet 1999; 64: 1541-1549.

6 Maichele AJ, Burwinkel B, Maire I, Sövik O, Kilimann MW: Mutations in the testis/liver isoform of the phosphorylase kinase $\gamma$ subunit (PHKG2) cause autosomal liver glycogenosis in the gsd rat and in humans. Nat Genet 1996; 14: 337-340.

7 Burwinkel B, Maichele AJ, Aagenaes Ö et al: Autosomal glycogenosis of liver and muscle due to phosphorylase kinase deficiency is caused by mutations in the phosphorylase kinase $\beta$ subunit (PHKB). Hum Mol Genet 1997; 6: 1109-1115.

8 Van den Berg IET, van Beurden EACM, de Klerk JBC et al Autosomal recessive phosphorylase kinase deficiency in liver, caused by mutations in the gene encoding the $\beta$ subunit (PHKB). Am J Hum Genet 1997; 61: 539-546.

9 Burwinkel B, Shiomi S, Al Zaben A, Kilimann MW: Liver glycogenosis due to phosphorylase kinase deficiency: PHKG2 gene structure and mutations associated with cirrhosis. Hum Mol Genet 1998; 7: 149-154.

10 Burwinkel B, Tanner MS, Kilimann MW: Phosphorylase kinase deficient liver glycogenosis: progression to cirrhosis in infancy associated with PHKG2 mutations (H144Y and L225R). J Med Genet 2000; 37: 376-377.

11 Abarbanel JM, Bashan N, Potashnik R, Osimani A, Moses SW, Herishanu Y: Adult muscle phosphorylase b kinase deficiency. Neurology 1986; 36: 560-562.

12 Clemens PR, Yamamoto M, Engel AG: Adult phosphorylase b kinase deficiency. Ann Neurol 1990; 28: 529-538.

13 Carrier H, Maire I, Vial C, Rambaud G, Flocard F, Flechaire A: Myopathic evolution of an exertional muscle pain syndrome with phosphorylase b kinase deficiency. Acta Neuropathol 1990; 81: 84-88.

14 Wilkinson DA, Tonin P, Shanske S, Lombes A, Carlson GM, DiMauro S: Clinical and biochemical features of 10 adult patients with muscle phosphorylase kinase deficiency. Neurology 1994; 44: 461-466.

15 Laforet P, Eymard B, Lombes A et al: Exercise intolerance caused by muscular phosphorylase kinase deficiency. Contribution of in vivo metabolic studies. Rev Neurol (Paris) 1996; 152: 458-464.

16 Bak H, Cordato D, Carey WF, Milder D: Adult-onset exercise intolerance due to phosphorylase $\mathrm{b}$ kinase deficiency. $J$ Clin Neurosci 2001; 8: 286-287.

17 Danon MJ, Carpenter S, Manaligod JR, Schliselfeld LH: Fatal infantile glycogen storage disease: deficiency of phosphofructokinase and phosphorylase b kinase. Neurology 1981; 31: 1303-1307.

18 Ohtani Y, Matsuda I, Iwamasa T, Tamari H, Origuchi Y, Miike T: Infantile glycogen storage myopathy in a girl with phosphorylase kinase deficiency. Neurology 1982; 32: 833-838.

19 Iwamasa T, Fukuda S, Tokumitsu S, Ninomiya N, Matsuda I, Osami M: Myopathy due to glycogen storage disease. Exp Mol Pathol 1983; 38: 405-420.

20 Shin YS, Plöchl E, Podskarbi T, Muss W, Pilz P, Puttinger R: Fatal arthrogryposis with respiratory insufficiency: a possible case of 
muscle phosphorylase b kinase deficiency. J Inher Metab Dis 1994; 17: 153-155.

21 Sahin G, Gungor T, Rettwitz-Volk W, Schlote W et al: Infantile muscle phosphorylase-b-kinase deficiency - a case report. Neuropediatrics 1998; 29: 48-50.

22 Bührer C, van Landeghem F, Brück W, Felderhoff-Müser U, Vorgerd M, Obladen M: Fetal-onset severe skeletal muscle glycogenosis associated with phosphorylase-b kinase deficiency. Neuropediatrics 2000; 31: 104-106.

23 Schneider A, Davidson JJ, Wüllrich A, Kilimann MW: Phosphorylase kinase deficiency in I-strain mice is associated with a frameshift mutation in the $\alpha$ subunit muscle isoform. Nat Genet 1993; 5: 381-385.

24 Wehner M, Clemens PR, Engel AG, Kilimann MW: Human muscle glycogenosis due to phosphorylase kinase deficiency associated with a nonsense mutation in the muscle isoform of the $\alpha$ subunit. Hum Mol Genet 1994; 3: 1983-1987.

25 Bruno C, Manfredi G, Andreu AL, Shanske S, Krishna S, Ilse WK, DiMauro S: A splice junction mutation in the $\alpha_{M}$ gene of phosphorylase kinase in a patient with myopathy. Biochem Biophys Res Commun 1998; 249: 648-651.

26 Lederer B, van Hoof F, van den Berghe G, Hers HG: Glycogen phosphorylase and its converter enzymes in haemolysates of normal human subjects and of patients with type VI glycogenstorage disease. Biochem J 1975; 147: 23-35.

27 Shin YS: Diagnosis of glycogen storage disease. J Inher Metab Dis 1990; 13: 419-434.

28 Wehner M, Kilimann MW: Human cDNA encoding the muscle isoform of the phosphorylase kinase $\gamma$ subunit (PHKG1). Hum Genet 1995; 96: 616-618.

29 Cawley KC, Akita CG, Angelos KL, Walsh DA: Characterization of the gene for rat phosphorylase kinase catalytic subunit. J Biol Chem 1993; 268: 1194-1200.

30 Maichele AJ, Farwell NJ, Chamberlain JS: A B2 repeat insertion generates alternate structures of the mouse muscle $\gamma$ phosphorylase kinase gene. Genomics 1993; 16: 139-149.

31 Wüllrich A, Hamacher C, Schneider A, Kilimann M: The multiphosphorylation domain of the phosphorylase kinase $\alpha_{M}$ and $\alpha_{\mathrm{L}}$ domains is a hotspot of differential mRNA processing and of molecular evolution. J Biol Chem 1993; 268: 23208-23214.

32 Wüllrich-Schmoll A, Kilimann MW: Structure of the human gene encoding the phosphorylase kinase $\beta$ subunit (PHKB). Eur $J$ Biochem 1996; 238: 374-380.

33 Fischer R, Koller M, Flura $\mathrm{M}$ et al: Multiple divergent mRNAs code for a single human calmodulin. J Biol Chem 1988; 263: 17055-17062.

34 Kubisch C, Wicklein EM, Jentsch TJ: Molecular diagnosis of McArdle's disease: revised genomic structure of the myophosphorylase gene and identification of a novel mutation. Hum Mutat 1998; 12: 27-32.

35 Vorgerd M, Kubisch C, Burwinkel B et al: Mutation analysis in myophosphorylase deficiency (McArdle's disease). Ann Neurol 1998; 43: 326-331.

36 Burwinkel B, Shin YS, Bakker HD et al: Mutation hotspots in the PHKA2 gene in X-linked liver glycogenosis due to phosphorylase kinase deficiency with atypical activity in blood cells. Hum Mol Genet 1996; 5: 653-658.

37 Burwinkel B, Moses SW, Kilimann MW: Phosphorylase-kinasedeficient liver glycogenosis with an unusual biochemical phenotype in blood cells associated with a missense mutation in the $\beta$ subunit gene $(P H K B)$. Hum Genet 1997; 101: 170-174.

38 Bruno C, Servidei S, Shanske S et al: Glycogen branching enzyme deficiency in adult polyglucosan body disease. Ann Neurol 1993; 33: 88-93.

39 Shen J, Bao Y, Liu HM, Lee P, Leonard JV, Chen YT: Mutations in exon 3 of the glycogen debranching enzyme gene are associated with glycogen storage disease type III that is differentially expressed in liver and muscle. J Clin Invest 1996; 98: 352-357.
40 Ziemssen F, Sindern E, Schröder JM et al: Novel missense mutations in the glycogen-branching enzyme gene in adult polyglucosan body disease. Ann Neurol 2000; 47: 536-540.

41 Harmann B, Zander NF, Kilimann MW: Isoform diversity of phosphorylase kinase $\alpha$ and $\beta$ subunits generated by alternative splicing. J Biol Chem 1991; 266: 15631-15637.

42 Ikeshima H, Yuasa S, Matsuo K, Kawamura K, Hata J, Takano T: Expression of three nonallelic genes coding calmodulin exhibits similar localization on the central nervous system of adult rats. J Neurosci Res 1993; 36: 111-119.

43 Bender PK, Dedman JR, Emerson CP: The abundance of calmodulin mRNAs is regulated in phosphorylase kinasedeficient skeletal muscle. J Biol Chem 1988; 263: 9733-9737.

44 Milan D, Jeon JT, Looft C et al: A mutation in PRKAG3 associated with excess glycogen content in pig skeletal muscle. Science 2000; 288: $1248-1251$.

45 Jones TA, da Cruz e Silva EF, Spurr NK, Sheer D, Cohen PTW: Localisation of the gene encoding the catalytic $\gamma$ subunit of phosphorylase kinase to human chromosome bands 7p12-q21. Biochim Biophys Acta 1990; 1048: 24-29.

46 Maichele AJ, Chamberlain JS: The $\gamma$ phosphorylase kinase gene, $\mathrm{Phkg}$, maps to mouse chromosome 5 near Gus. Mammalian Genome 1994; 5: 15-18.

47 Davidson JJ, Özcelik T, Hamacher C, Willems PJ, Francke U, Kilimann MW: cDNA cloning of a liver isoform of the phosphorylase kinase $\alpha$ subunit and mapping of the gene to Xp22.2-p22.1, the region of human X-linked liver glycogenosis. Proc Natl Acad Sci USA 1992; 89: 2096-2100.

48 Calalb MB, Fox DT, Hanks SK: Molecular cloning and enzymatic analysis of the rat homolog of "PhK- $\gamma \mathrm{T}$ ", an isoform of phosphorylase kinase catalytic subunit. J Biol Chem 1992; 267: 1455-1463.

49 Westphal V, Kjaergaard S, Schollen E et al: A frequent mild mutation in ALG6 may exacerbate the clinical severity of patients with congenital disorder of glycosylation Ia (CGD-Ia) caused by phosphomannomutase deficiency. Hum Mol Genet 2002; 11: 599-604.

50 Burwinkel B, Sanjad SA, Al-Sabban E, Al-Abbad A, Kilimann MW: A mutation in GLUT2, not in phosphorylase kinase subunits, in hepato-renal glycogenosis with Fanconi syndrome and low phosphorylase kinase activity. Hum Genet 1999; 105: 240-243.

51 Halestrap AP, Price NT: The proton-linked monocarboxylate transporter (MTC) family: structure, function and regulation. Biochem J 1999; 343: 281-299.

52 Zorzano A, Fandos C, Palacin M: Role of plasma membrane transporters in muscle metabolism. Biochem J 2000; 349: 667-688.

53 Wang ND, Finegold MJ, Bradley A et al: Impaired energy homeostasis in C/EBP alpha knockout mice. Science 1995; 269: 1108-1112.

54 Croniger CM, Millward C, Yang J et al: Mice with a deletion in the gene for CCAAT/enhancer-binding protein beta have an attenuated response to cAMP and impaired carbohydrate metabolism. J Biol Chem 2001; 276: 629-638.

55 Lerin C, Montell E, Berman HK, Newgard CB, Gomez-Foix AM: Overexpression of protein targeting to glycogen in cultured human muscle cells stimulates glycogen synthesis independent of glycogen and glucose 6-phosphate levels. J Biol Chem 2000; 275: 39991-39995.

56 Cawley KC, Akita CG, Wineinger MA, Carlsen RC, Gorin FA, Walsh DA: Coordinated expression of phosphorylase kinase subunits in regenerating skeletal muscle. I Biol Chem 1992; 267 17287-17295

$57 \mathrm{Ng}$ DC, Carlsen RC, Walsh DA: Neural regulation of the formation of skeletal muscle phosphorylase kinase holoenzyme in adult and developing rat muscle. Biochem $J$ 1997; 325: 793-800. 